\title{
DETEÇÃO DE SINAIS TCM EM CANAIS COM INTERCALAÇÃO E DESVANECIMENTO NÃO-SELETIVO EM FREQÜÊNCIA
}

\author{
Tomie Sugahara e Raimundo Sampaio Neto \\ Pontifícia Universidade Católica - CETUC \\ Rua Marquês de São Vicente, 225 \\ Gávea, Rio de Janeiro, RJ, 22453-900 \\ E-mail: raimundo@cetuc-puc-rio.br
}

\begin{abstract}
Resumo - Neste artigo examinamos uma nova estratégia para deteção de sinais com modulação codificada em treliça, transmitidos através de um canal com desvanecimento de Rayleigh não-seletivo em frequência. $O$ algoritmo de Viterbi é usado em sistemas com e sem intercalação para escolher a sequência codificada que minimiza uma métrica aditiva que incorpora uma estimativa da amostra do processo de desvanecimento em cada intervalo de símbolo. Estimadores de Kalman atualizam essas estimativas em um processamento por percurso sobrevivente, gerando, em cada instante, uma estimativa distinta por nó da treliça. Curvas de taxa de erro de bit, levantadas através de simulações no computador, são comparadas às curvas obtidas com conhecimento ideal do desvanecimento $\mathrm{e}$ às curvas relativas a outros métodos na literatura.
\end{abstract}

\begin{abstract}
We look into a new strategy for detecting signals with trellis coded modulation transmitted over a frequencynonselective fading channel. For both, interleaved and noninterleaved systems, the Viterbi algorithm is used to choose the coded sequence which minimizes an additive metric incorporating estimates of the fading samples in each symbol interval. Kalman estimators are used to recursively update these estimates on a per survivor basis whereupon, at a given instant, a distinct estimate is associated to each trellis node. Bit error rate curves evaluated by computer simulations are compared to curves obtained with ideal fading knowledge and to curves pertaining to other methods in the literature.
\end{abstract}

Palavras chaves: TCM, desvanecimento de Rayleigh, algoritmo de Viterbi, estimação de canal, processamento por percurso sobrevivente.

\section{INTRODUÇÃO}

A deteção coerente de sequências equiprováveis de sinais com modulação PSK codificada em treliça (TCM), transmitidas através de um canal com ruído gaussiano branco aditivo e com desvanecimento de Rayleigh nãoseletivo em frequência, tem sido o objeto de muitos artigos publicados recentemente [7] [9]. Vários graus de intercalação do canal tem sido usados, levando-se em conta o tempo de coerência do desvanecimento e o atraso tolerável. Em geral, o critério de detecção de sequiência de Máxima Verossimilhança é adotado, dada a disponibilidade, na recepção, de estimativas das amostras do desvanecimento. A geração dessas estimativas tem suscitado soluções diversificadas. Como canais com desvanecimento não-seletivo em frequência introduzem uma distorção multiplicativa variante no tempo no sinal de banda-básica transmitido através dele, então a observação dessa distorção depende da remoção do sinal transmitido, o que no caso da modulação PSK é conseguido multiplicando-se a amostra do sinal recebido pelo complexo conjugado do sinal transmitido, suposto com energia unitária. Porém, o sinal transmitido não é conhecido na recepção, logo esse procedimento, chamado de remoção de modulação, requer uma estimativa do sinal transmitido. Um esquema [6] que dispensa essa estimativa consiste em multiplexar uma sequiência de símbolos conhecidos com a seqüência de símbolos de dados transmitidos e usar somente as amostras do sinal recebido correspondentes aos símbolos conhecidos para obter observações periódicas da distorção. Da interpolação dessas observações são obtidas as estimativas da distorção nos intervalos de transmissão dos dados aleatórios. As desvantagens desse esquema estão no gasto de energia com os símbolos conhecidos e no aumento da ineficiência de transmissão para atender à redução do tempo de coerência do canal. Esquemas mais eficientes [7] [9] derivam observações também das amostras do sinal recebido relativas a dados aleatórios, o que nos remete de volta à questão da remoção de modulação. A estimativa do sinal transmitido usada para esse fim, geralmente difere da decisão final fornecida pelo decodificador de Viterbi sendo chamada de decisão tentativa.

Na ausência de intercalação, decisões preliminares na saída do decodificador de Viterbi podem servir como decisões tentativas. Uma decisão tentativa do $(\mathrm{k}-\delta)$-ésimo símbolo transmitido é obtida retrocedendo $\delta$ transições ao longo do percurso sobrevivente do estado com a menor métrica acumulada no k-ésimo instante [1]. Geralmente, quanto maior o atraso $\delta$, mais confiável a decisão preliminar, embora menor a correlação entre a observação resultante da remoção de modulação usando essa decisão tentativa e a amostra da distorção no k-ésimo intervalo, o que pode resultar em uma estimativa ruim. Ademais, a mesma estimativa sendo usada no cálculo das métricas de todos os ramos da treliça na k-ésima transição causa uma realimentação de erros de decisão no algoritmo de decodificação. No esquema proposto neste artigo, no késimo instante, obtém-se uma estimativa de distorçāo por percurso sobrevivente, usando os sinais desse percurso 
como decisões tentativas [2]. Com isso, eliminam-se as duas possíveis fontes de erro do método em [1]: 0 atraso $\delta$ passa a ser nulo e a realimentação de erro afeta apenas o percurso sobrevivente no qual o erro ocorreu.

Com o uso de intercalação, a sequiência de amostras do sinal recebido na entrada do decodificador de Viterbi contém símbolos de dados na ordem de saída do codificador multiplicados por amostras de distorção fora da ordem de suas ocorrências no canal de transmissão. Isso cria dificuldades para a aplicação da decodificação com estimação da distorção por percurso sobrevivente porque em cada instante, as estimativas necessárias para calcular as métricas de transição (1) não podem vir da atualização das estimativas usadas na transição anterior (2) podem precisar vir da atualização de estimativas a serem usadas em uma transição futura. No esquema proposto neste artigo, estimativas obtidas em transições passadas são recuperadas retrocedendo ao longo dos percursos sobreviventes enquanto amostras de distorção que ocorrem em transições futuras mas de cujas estimativas depende o cálculo das métricas na transição presente são estimadas pelo método de inserção de símbolos conhecidos em [6].

Um esquema comum na literatura para canais com intercalação, é o receptor de dois estágios [7] [9], onde no primeiro estágio, antes do desintercalador, são obtidas estimativas de distorção a partir de decisões tentativas geradas por um algoritmo de detecção de símbolos nãocodificados. Além da ajuda de símbolos conhecidos para evitar um comportamento errático, estes algoritmos no primeiro estágio usam estimativas tentativas da distorção, ditando um processamento redundante para decisões $e$ estimativas de distorção.

O conteúdo deste artigo está organizado da seguinte forma: na Seção 2 definimos o modelo do sistema; os dois novos esquemas são descritos na Seção 3 e resultados de simulação apresentados na Seção 4.

\section{MODELO DO SISTEMA}

A Fig. 1 mostra o diagrama de blocos do sistema de banda básica.

O sinal de entrada para o codificador-modulador em treliça é uma sequiência de dígitos binários de dados e o de saída, uma sequiência de símbolos PSK codificados e com energia unitária. Em sistema sem intercalação, essa seqüência codificada segue diretamente para um filtro de formatação de pulso com resposta impulsiva $h_{T X}(t)=g(t)$ com energia unitária. Em sistema com intercalação, antes de ser entregue ao filtro, a sequiência codificada passa pelo intercalador e, em seguida, pelo multiplexador, onde sofre a inserção de símbolos conhecidos à taxa de 1 símbolo conhecido para cada L-1 símbolos codificados. Por facilitar a descrição dos algoritmos propostos, o método de intercalação adotado neste artigo é o de bloco, com escrita por linhas e leitura por colunas no bloco do intercalador e o inverso no do desintercalador. $\mathrm{Na}$ saída do filtro, o sinal de banda básica pode ser escrito como $a^{\prime}(t)=\sum_{m} a^{\prime}[m] g(t-m T)$ onde $1 / T$ é a taxa de símbolos.

O sinal $a^{\prime}(t)$ é transmitido através de um canal com desvanecimento não-seletivo em frequência e com ruído aditivo, produzindo na entrada do receptor:

$r^{\prime}(t)=y(t) a^{\prime}(t)+w(t)$

O desvanecimento $y(t)$ e o ruído aditivo $w(t)$ são processos Gaussianos complexos, estacionários e independentes entre si, com partes real e imaginária independentes e identicamente distribuídas. Ambos os processos têm média nula, sendo a variância de $y(t)$ igual a 1 e a função de densidade espectral de potência de $w(t)$ constante e igual a $\sigma_{n}^{2}$.

Se $y(t)$ for lento o suficiente para poder ser considerado constante durante um intervalo de símbolo e se $g(t)$ satisfizer o critério de Nyquist para interferência intersimbólica nula, então o componente de sinal de $r^{\prime}(t)$ pode ser escrito como $y(t) a^{\prime}(t) \cong \sum_{m} y[m] a^{\prime}[m] g(t-m T)$ onde y[m] é o valor de $y(t)$ durante o m-ésimo intervalo. Para $\int_{m T}^{(m+1) T}|g(t-m T)|^{2} d t \cong 1$, a energia média recebida por símbolo pode ser calculada como $E_{s}=E\left\{\int_{m T}^{(m+1) T}\left|y[m] a^{\prime}[m] g(t-m T)\right|^{2} d t\right\} \cong 1$

O sinal recebido $r^{\prime}(t)$ passa por um filtro casado com uma resposta impulsiva $h_{R X}(t)=g(T-t)$. Amostrando a saída do filtro a cada $\mathrm{T}$ segundos, obtém-se no instante $\mathrm{t}=(\mathrm{k}+1) \mathrm{T}$ :

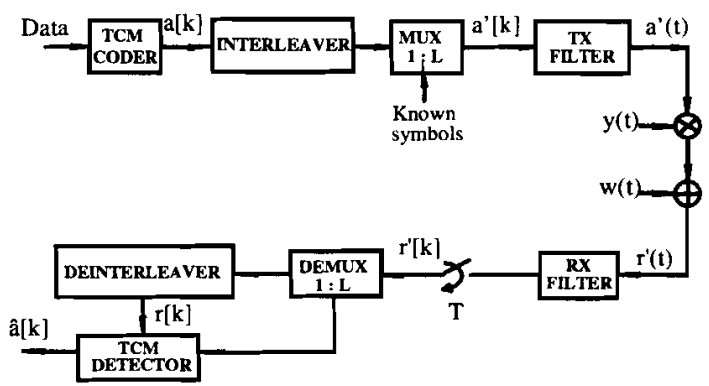

Fig. 1. Diagrama de blocos do sistema de banda básica.

$\left.r^{\prime}[k]=y[k] a^{\prime}[k]\right]_{-\infty}^{\infty} g^{2}(\tau-k T) d \tau+$
$\left.+\sum_{m \neq k} y[m] a^{\prime}[m]\right]_{-\infty}^{\infty} g(\tau-m T) g(\tau-k T) d \tau+n[k]$

Para $\int_{-\infty}^{\infty} g(\tau-m T) g(\tau-k T) d \tau=\delta((m-k) T)$ tem-se: 
$r^{\prime}[k]=y[k] a^{\prime}[k]+n[k]$

As amostras de ruído $n[k]=\int_{-\infty}^{\infty} w(\tau) g(\tau-k T) d \tau$ são variáveis aleatórias Gaussianas, complexas, com média nula, variância $\sigma_{n}^{2}$ e independentes entre si. As partes real e imaginária de cada variável são independentes $e$ identicamente distribuídas.

Em sistema com intercalação, as amostras do sinal recebido seguem para o demultiplexador, de onde as amostras relativas a símbolos conhecidos são enviadas diretamente para o detetor TCM enquanto as amostras relativas a símbolos de dados passam antes pelo desintercalador. Em sistema sem intercalação, o demultiplexador e o desintercalador não estão presentes e as amostras do sinal recebido seguem diretamente para $o$ detetor. $O$ algoritmo implementado no bloco do detetor TCM é descrito na Seção 3 para sistemas com e sem intercalação.

\section{DECODIFICAÇÃO TCM COM ESTIMAÇÃO POR PERCURSO SOBREVIVENTE}

\section{Preliminares}

Seguindo a notação na Fig. 1, sejam os vetores ${ }^{1}$ aleatórios $\mathbf{a}=(\mathrm{a}[0], \ldots, \mathrm{a}[\mathrm{k}], . .)^{t}$ de símbolos na saída do codificador $\mathrm{e}$ $\mathbf{r}=(\mathrm{r}[0], \ldots, \mathrm{r}[\mathrm{k}], . .)^{\mathrm{t}}$ de amostras do sinal recebido na entrada do decodificador. $O$ vetor de amostras do desvanecimento na mesma ordem cronológica com que comparecem em $\mathbf{r}$ é denotado por $\mathbf{y}^{\prime}=\left(y^{\prime}[0], ., y^{\prime}[k], . .\right)^{t}$ e é estimado pelo vetor aleatório $\hat{\mathbf{y}}^{\prime}$. Supondo $\hat{\mathbf{y}}^{\prime}$ e a estatisticamente independentes e todas as realizações de a igualmente prováveis, o detetor de máxima probabilidade $a$ posteriori escolhe o $\mathbf{a}_{\mathrm{j}}$ que maximiza $\mathrm{p}_{\mathbf{r}}\left(\mathbf{R} \mid \mathbf{a}=\mathbf{a}_{\mathrm{j}}, \hat{\mathbf{y}}^{\prime}=\hat{\mathbf{Y}}^{\prime}\right)$.

Se $\mathbf{y}^{\prime}$ e $\hat{\mathbf{y}}^{\prime}$ forem conjuntamente gaussianos de médias nulas, pode-se mostrar que, condicionado aos eventos especificados, $\mathbf{r}$ é um vetor gaussiano de média $\mu_{j}=\mathbf{A}_{j} \Re_{y^{\prime} \hat{\mathbf{y}}} \Re_{\hat{\mathbf{y}}}^{-1} \hat{\mathbf{Y}}^{\prime}$ e matriz de covariância

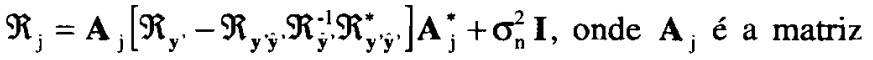
diagonal com os elementos de $\mathbf{a}_{\mathbf{j}}$ na diagonal, $\mathbf{I}$ é a matriz identidade, $\Re_{y^{\prime}}$ e $\Re_{\hat{y}}$, são as matrizes de covariância de $\mathbf{y}^{\prime} \mathrm{e}$ $\hat{\mathbf{y}}^{\prime}$, respectivamente, e $\Re_{y^{\prime} \hat{y}^{\prime}}$ é a matriz de covariância cruzada desses mesmos vetores. Logo, a regra de decisão consiste em escolher o $a_{j}$ que minimiza a métrica abaixo:

$\mathbf{M}_{\mathrm{j}}=\left(\mathbf{R}-\mu_{\mathrm{j}}\right)^{*} \Re_{\mathrm{j}}^{-1}\left(\mathbf{R}-\mu_{\mathrm{j}}\right)+\ln \operatorname{det}\left(\Re_{\mathrm{j}}^{-1}\right)$

\footnotetext{
'Notação: ' denota transposição $\mathrm{e}^{*}$ denota conjugação complexa seguida de transposição.
}

Para que a regra de decisão seja baseada na métrica euclidiana

$\mathbf{M}_{\mathrm{j}}=\sum_{\mathbf{k}}\left|\mathrm{R}[\mathrm{k}]-\mu_{\mathrm{j}}[\mathrm{k}]\right|^{2}$

$\Re_{\mathrm{j}}$ deve ser diagonal, como ocorre para:

1. conhecimento ideal do desvanecimento

2. sistema com intercalação infinita

No primeiro caso, $\hat{\mathbf{y}}^{\prime}=\mathbf{y}^{\prime}$ acarreta $\Re_{\mathbf{y}^{\prime}}=\Re_{\hat{\mathbf{y}}},=\Re_{\mathbf{y}^{\prime}}$ logo $\Re_{\mathrm{j}}=\sigma_{\mathrm{n}}^{2} \mathbf{I}$. No segundo caso, os componentes de $\mathbf{y}^{\prime}$ e de $\hat{\mathbf{y}}^{\prime}$ são independentes. Logo $\Re_{y \hat{y}}$ e $\Re_{j}$ são diagonais.

\section{Notação}

Seja um código TCM com $\mathrm{S}$ estados. No instante $\mathrm{kT}$, o nó da treliça correspondente ao i-ésimo estado, $i \in\{0,1, \ldots, S-1\}$, é denotado por $\Delta_{k}^{i}$. O ramo da treliça entre os nós $\Delta_{k}^{\mathrm{i}}$ e $\Delta_{\mathbf{k}+1}^{\mathrm{m}}$ é denotado por $\operatorname{arc}\left(\Delta_{\mathbf{k}}^{\mathrm{i}}, \Delta_{\mathbf{k}+1}^{\mathrm{m}}\right)$ e o símbolo TCM associado a ele, por a $\left(\Delta_{\mathrm{k}}^{\mathrm{i}}, \Delta_{\mathrm{k}+1}^{\mathrm{m}}\right)$. Na decodificação, o percurso sobrevivente associado ao nó $\Delta_{k}^{\mathrm{i}}$ é denotado por $\mathbf{p}\left(\Delta_{\mathrm{k}}^{\mathrm{i}}\right)$ e o nó desse percurso no instante jT, por $\Delta_{\mathrm{j}}^{\mathrm{p}\left(\Delta_{\mathrm{k}}^{\mathrm{i}}\right)}$, $\mathrm{j}=0,1, \ldots, \mathrm{k}-1$. Na Fig. 2, para um código de 2 estados, indicamos em negrito o percurso sobrevivente $\mathbf{p}\left(\Delta_{3}^{0}\right)$ associado ao nó $\Delta_{3}^{0}$ e usamos a notação acima para os demais nós desse percurso

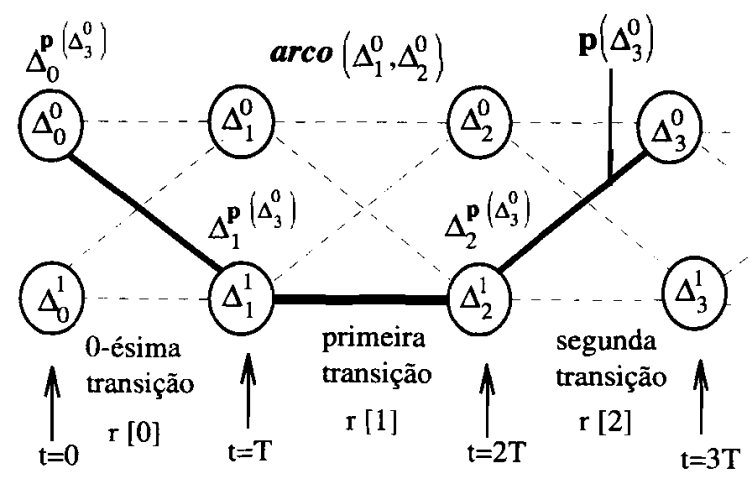

Fig. 2. Exemplo de notação, código de 2 estados.

\section{Intróito}

Nos algoritmos propostos nesta seção, a cada nó no instante $\mathrm{kT}, \Delta_{\mathrm{k}}^{\mathrm{i}}$, são associados:

1. um percurso sobrevivente, $\mathbf{p}\left(\Delta_{\mathrm{k}}^{\mathrm{i}}\right)$

2. a métrica acumulada desse percurso, $\Gamma\left(\Delta_{\mathrm{k}}^{\mathrm{i}}\right)$

3. no caso de um estimador de Kalman, um vetor de estimativa e a matriz de covariância do erro de estimação. 
Vamos supor que os três itens especificados acima já estejam associados aos nós no instante $\mathrm{kT}$. $\mathrm{O}$ algoritmo de Viterbi determina que, para chegar ao instante $(\mathrm{k}+1) \mathrm{T}$, devese estender de um ramo todos os percursos sobreviventes do instante $\mathrm{kT}$, calcular as métricas acumuladas dos percursos estendidos e para cada nó $\Delta_{\mathrm{k}+1}^{\mathrm{m}}$ no instante $(\mathrm{k}+1) \mathrm{T}$, selecionar o percurso estendido com a menor métrica acumulada como o percurso sobrevivente desse nó.

A métrica de transição do ramo associado ao símbolo $a_{j}[\mathrm{k}]$ é $\left|R[k]-a_{j}[k] \hat{Y}^{\prime}[k]\right|^{2}$, onde $\hat{Y}^{\prime}[k]$ é uma estimativa da amostra do desvanecimento em $\mathrm{R}[\mathrm{k}]$. Se o processo das amostras, $\{\mathrm{y}[\mathrm{k}]\}$, for um processo de Média Móvel Auto Regressivo, um filtro de Kalman pode ser obtido [2] que fornece $\hat{\mathbf{y}}_{\mathbf{k}}=\mathrm{E}\left\{\mathbf{y}_{\mathbf{k}} \mid \mathbf{z}_{\mathrm{k}}, \hat{\mathbf{y}}_{\mathrm{k}-1}\right\}$ onde $\mathbf{z}_{\mathrm{k}}$ é um vetor de observações do processo, obtido a partir da remoção de modulação das amostras do sinal recebido; $\hat{\mathbf{y}}_{\mathrm{k}-1}$ é o vetor passado e $\hat{\mathbf{y}}_{\mathbf{k}}$ o vetor atualizado de estimativas das amostras do processo. Analogamente, um preditor de 1 passo de Kalman pode ser obtido [2] que fornece $\hat{\mathbf{y}}_{\mathbf{k}}=\mathrm{E}\left\{\mathbf{y}_{\mathbf{k}} \mid \mathbf{z}_{\mathrm{k}-1}, \hat{\mathbf{y}}_{\mathbf{k}-1}\right\}$. Para um processo de ordem N, todos esses vetores têm $\mathrm{N}$ componentes cujos índices cronológicos crescem até o máximo indicado no subscrito do nome do vetor. Assim, $\hat{\mathbf{y}}_{\mathbf{k}}=(\hat{\mathrm{y}}[\mathrm{k}-\mathrm{N}+1], ., \hat{\mathrm{y}}[\mathrm{k}])^{\mathrm{t}} \quad$ enquanto $\hat{\mathbf{y}}_{\mathrm{k}-1}=(\hat{\mathrm{y}}[\mathrm{k}-\mathrm{N}], ., \hat{\mathrm{y}}[\mathrm{k}-1])^{\mathrm{t}}$.

Denotamos por $\mathbf{Y}$ e $\mathbf{Y}^{\prime}$ aos vetores de amostras do desvanecimento na ordem em que aparecem nos vetores $\mathbf{R}^{\prime}$ de amostras do sinal recebido na saída do amostrador e $\mathbf{R}$ de amostras do sinal recebido na entrada do decodificador, respectivamente. A estratégia de decodificação com estimação do desvanecimento por percurso sobrevivente não oferece dificuldades em sistema sem intercalação porque os componentes do vetor $\mathbf{R}$, na entrada do decodificador, contêm símbolos na ordem em que saíram do codificador e amostras de distorção na ordem em que foram geradas no canal, isto é, $\mathbf{Y}^{\prime}=\mathbf{Y}$. Essa facilidade não existe em sistema com intercalação porque os componentes de $\mathbf{R}$ contêm símbolos na ordem em que saíram do codificador mas amostras do desvanecimento fora de sua ordem de geração no canal, isto é, $\mathbf{Y}^{\prime} \neq \mathbf{Y}$.

\section{Sistema sem Intercalação}

No caso de um preditor de 1 passo, o vetor de estimativa $\hat{\mathbf{Y}}_{\mathrm{k}}\left(\Delta_{\mathrm{k}}^{\mathrm{i}}\right)$, associado ao nó $\Delta_{\mathrm{k}}^{\mathrm{i}}$, contém a estimativa de $\mathrm{Y}^{\prime}[\mathrm{k}]$ necessária para calcular as métricas de transição de todos os ramos que partem desse nó. Para cada nó $\Delta_{k+1}^{\mathrm{m}}$ no instante $(\mathrm{k}+1) \mathrm{T}$, uma vez determinado o seu percurso sobrevivente, $\mathbf{p}\left(\Delta_{\mathbf{k}+1}^{\mathrm{m}}\right)$, atualiza-se $\mathbf{o}$ vetor de estimativa anterior, $\hat{\mathbf{Y}}_{\mathrm{k}}\left(\Delta_{\mathrm{k}}^{\mathrm{p}\left(\Delta_{\mathrm{k}+1}^{\mathrm{m}}\right)}\right)$, baseado no vetor de observações $\mathbf{Z}_{\mathrm{k}}$, obtido usando os símbolos de $\mathbf{p}\left(\Delta_{\mathbf{k}+1}^{\mathrm{m}}\right)$ para tentativamente remover a modulação das amostras do sinal recebido. $O$ valor desse vetor atualizado, $\hat{\mathbf{Y}}_{\mathbf{k}+1}\left(\Delta_{\mathbf{k}+1}^{\mathrm{m}}\right)=\mathrm{E}\left\{\mathbf{y}_{\mathbf{k}+1} \mid \mathbf{Z}_{\mathbf{k}}, \hat{\mathbf{Y}}_{\mathbf{k}}\left(\Delta_{\mathbf{k}}^{\mathrm{p}\left(\Delta_{\mathrm{k}+1}^{\mathrm{m}}\right)}\right)\right\}$, é associado ao nó $\Delta_{\mathrm{k}+1}^{\mathrm{m}}$.

No caso de um filtro, para obter as métricas de todos os ramos que saem do nó $\Delta_{\mathrm{k}}^{\mathrm{i}}$ no instante $\mathrm{kT}$, primeiro atualizase o vetor de estimativa $\hat{\mathbf{Y}}_{\mathrm{k}-1}\left(\Delta_{\mathrm{k}}^{\mathrm{i}}\right)$ associado a esse nó, obtendo um vetor $\hat{\mathbf{Y}}_{\mathbf{k}}\left(\Delta_{\mathrm{k}}^{\mathrm{i}}, \Delta_{\mathrm{k}+1}^{\mathrm{m}}\right)=\mathrm{E}\left\{\mathbf{y}_{\mathrm{k}} \mid \mathbf{Z}_{\mathrm{k}}\left(\Delta_{\mathrm{k}}^{\mathrm{i}}, \Delta_{\mathrm{k}+1}^{\mathrm{m}}\right), \hat{\mathbf{Y}}_{\mathrm{k}-1}\left(\Delta_{\mathrm{k}}^{\mathrm{i}}\right)\right\} \quad$ para cada ramo $\operatorname{arc}\left(\Delta_{\mathrm{k}}^{\mathrm{i}}, \Delta_{\mathrm{k}+1}^{\mathrm{m}}\right)$ saindo de $\Delta_{\mathrm{k}}^{\mathrm{i}}$. O vetor de observações $\mathbf{Z}_{\mathbf{k}}\left(\Delta_{\mathrm{k}}^{\mathrm{i}}, \Delta_{\mathrm{k}+1}^{\mathrm{m}}\right)$, usado na atualização da estimativa para $\mathbf{o}$ ramo $\operatorname{arc}\left(\Delta_{k}^{i}, \Delta_{k+1}^{m}\right)$, é obtido tendo como decisões tentativas na "remoção" de modulação o símbolo $\mathrm{a}\left(\Delta_{\mathrm{k}}^{\mathrm{i}}, \Delta_{\mathrm{k}+1}^{\mathrm{m}}\right)$ associado a esse ramo $\mathrm{e}$ os símbolos pertencentes ao percurso sobrevivente $\mathbf{p}\left(\Delta_{\mathrm{k}}^{\mathrm{i}}\right)$. Para cada nó $\Delta_{\mathbf{k}+1}^{\mathrm{m}}$ no instante $(\mathrm{k}+1) \mathrm{T}$, uma vez obtido o seu percurso sobrevivente $p\left(\Delta_{k+1}^{m}\right)$, o vetor de estimativa que fica associado a esse nó é o vetor atualizado antes associado ao ramo do seu percurso sobrevivente, ou seja, $\quad \hat{\mathbf{Y}}_{k}\left(\Delta_{k+1}^{\mathrm{m}}\right)=\hat{\mathbf{Y}}_{\mathbf{k}}\left(\Delta_{\mathrm{k}}^{\mathrm{p}\left(\Delta_{k+1}^{\mathrm{m}}\right)}, \Delta_{\mathrm{k}+1}^{\mathrm{m}}\right)$.

\section{Sistema com Intercalação}

Devido à natureza modular da intercalação de bloco, assume-se que os indices de $\mathbf{Y}^{\prime}$ e de $\mathbf{Y}$ variam de 0 a LC -1 onde $\mathrm{L}$ e $C$ são os números de linhas e de colunas do bloco, respectivamente. Se chamarmos de $\mathrm{f}: \mathbf{Y}^{\prime} \rightarrow \mathbf{Y}$ e de $\mathbf{f}^{-1}: \mathbf{Y} \rightarrow \mathbf{Y}^{\prime}$ às funções de intercalação e de desintercalação, respectivamente, então a condição para que $\mathrm{Y}^{\prime}[\alpha]=\mathrm{f}^{-1}(\mathrm{Y}[\beta])$ possa ser recursivamente estimado é a existência das estimativas de $\mathrm{f}^{-1}(\mathrm{Y}[\mathrm{m}], \beta-\mathrm{N} \leq \mathrm{m} \leq \beta-1$. Pode-se mostrar que essa condição não é atendida para a primeira linha do bloco o que pode ser melhor entendido através do exemplo a seguir onde invocamos um "gênio" para fornecer as estimativas em falta. Explicamos também como as demais estimativas são obtidas usando a estratégia do processamento por percurso sobrevivente.

Para um bloco de tamanho $4 \times 4$, a função de desintercalação $\mathrm{f}^{-1}: \mathbf{Y} \rightarrow \mathbf{Y}^{\prime}$ fornece $\mathrm{Y}^{\prime}[0]=\mathrm{Y}[0]$, $\mathrm{Y}^{\prime}[1]=\mathrm{Y}[4], \mathrm{Y}^{\prime}[2]=\mathrm{Y}[8], \mathrm{Y}^{\prime}[3]=\mathrm{Y}[12]$, etc. Na Fig. 3 mostramos a posição, dentro do bloco desintercalador, dos componentes de $\mathbf{R}$, com indicação dos símbolos e dos componentes de $\mathbf{Y}$ contidos nos respectivos componentes de R. Como o envio dessas amostras para o decodificador é feito por linhas, a posição das amostras na treliça de decodificação fica conforme mostrado na Fig. 4. 


\begin{tabular}{|c|c|c|c|}
\hline $\left.\begin{array}{l}A[0] \\
y[0]\end{array}\right\} R[0]$ & $\left.\begin{array}{l}A[1] \\
y[4]\end{array}\right\} R[1]$ & $\left.\begin{array}{c}\mathrm{A}[2] \\
\mathrm{y}[8]\end{array}\right\}_{\mathrm{R}[2]}$ & $\left.\begin{array}{l}A[3] \\
y[12]\end{array}\right\}$ \\
\hline 3 & $\begin{array}{l}x[5] \\
y[5]\end{array}$ & $\begin{array}{l}y[9] \\
\}[6]\end{array}$ & $y[13]$ \\
\hline$y[2]\}^{R}$ & $\left.\begin{array}{c}y[6] \\
y[6]\end{array}\right\}$ & $\left.\begin{array}{l}A[10] \\
y[10]\end{array}\right\} R[10]$ & ] \\
\hline [3] $\}$ & 13] & 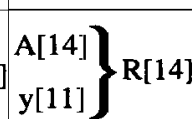 & $y[$ \\
\hline
\end{tabular}

Fig. 3. Posição dos símbolos e das amostras de desvanecimento no bloco desintercalador $4 \times 4$.

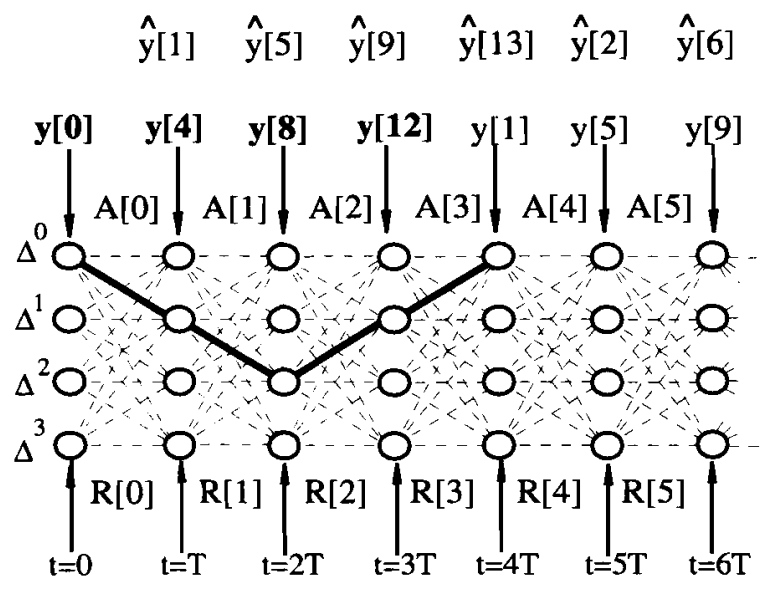

Fig. 4. Decodificação com estimação por percurso sobrevivente, código de 4 estados, bloco $4 \times 4$

Na Fig.4, em cada instante indicamos o componente de $\mathbf{Y}$ cuja estimativa é necessária para calcular as métricas dos ramos na transição seguinte. A estimativa de $\mathrm{Y}^{\prime}[0]=\mathrm{Y}[0]$ pode ser obtida da decodificação do bloco anterior, mas os componentes $\mathrm{Y}^{\prime}[1]=\mathrm{Y}[4], \mathrm{Y}^{\prime}[2]=\mathrm{Y}[8]$ e $\mathrm{Y}^{\prime}[3]=\mathrm{Y}[12]$, em negrito, não podem ser estimados recursivamente porque no caso de $Y^{\prime}[1]=Y[4]$, por exemplo, $Y^{\prime}[12]=f^{-1}(Y[3])$, $\mathrm{Y}^{\prime}[8]=\mathrm{f}^{-1}(\mathrm{Y}[2])$ e $\mathrm{Y}^{\prime}[4]=\mathrm{f}^{-1}(\mathrm{Y}[1])$ estão contidos em amostras do sinal recebido que ainda não foram decodificados. Considerando predição de 1 passo de Kalman, vamos supor, por ora, que um "gênio" forneça os valores corretos dos vetores de amostras $\mathbf{Y}_{0}, \mathbf{Y}_{4}, \mathbf{Y}_{8}$ e $\mathbf{Y}_{12}$ que ficam associados a todos os nós nos instantes $0,4 \mathrm{~T}, 8 \mathrm{~T}$ e $12 \mathrm{~T}$, respectivamente. Então, para cada nó $\Delta_{0}^{\mathrm{i}}$ no instante 0 , as métricas de todos os ramos saindo desse nó podem ser calculadas usando $\mathrm{Y}[0]$, contido em $\mathbf{Y}_{0}$. O próximo passo é, para cada nó $\Delta_{1}^{\mathrm{m}}$ no instante $\mathrm{T}$, uma vez determinado o seu percurso sobrevivente, $\mathbf{p}\left(\Delta_{1}^{\mathrm{m}}\right)$, atualizar o vetor $\mathbf{Y}_{0}$, baseado no vetor de observações $Z_{0}$, obtido usando o símbolo de
$\mathbf{p}\left(\Delta_{1}^{\mathrm{m}}\right)$ para "remover" a modulação de $\mathrm{R}[0]$. Os demais componentes de $Z_{0}$ podem ser obtidos de $Y_{0}$. $O$ vetor de predição atualizado, $\hat{\mathbf{Y}}_{1}\left(\Delta_{1}^{\mathrm{m}}\right)=\mathrm{E}\left\{\mathbf{y}_{1} \mid \mathbf{Z}_{0}, \mathbf{Y}_{0}\right\}$, fica associado ao nó $\Delta_{1}^{\mathrm{m}}$. O mesmo procedimento é repetido nos instantes $\mathrm{T}, 2 \mathrm{~T}$ e $3 \mathrm{~T}$, mutatis mutandis. No instante $4 \mathrm{~T}$, observa-se que a estimativa requerida do componente $Y^{\prime}[4]=Y[1]$ já está associada aos nós no instante T. Logo, para cada nó $\Delta_{4}^{\mathrm{j}}$ no instante atual, obtém-se essa estimativa, retrospectivamente, no nó $\Delta_{1}^{\mathrm{p}\left(\Delta_{4}^{\mathrm{j}}\right)}$ pertencente ao percurso sobrevivente de $\Delta_{4}^{\mathrm{j}}$. Na Fig.4 um exemplo para o percurso sobrevivente do nó $\Delta_{4}^{0}$ está mostrado em negrito, indicando que a estimativa desejada está associada ao nó $\Delta_{1}^{1}$. O procedimento de atualização de estimativa já foi descrito e a decodificação prossegue dessa forma nos intervalos seguintes.

Quanto ao problema do "gênio", há várias maneiras de prover as estimativas que não podem ser obtidas recursivamente e da solução adotada depende o desempenho desta estratégia. Os resultados de simulação na Seção 4 foram obtidos multiplexando uma sequência de símbolos conhecidos com a sequência intercalada de símbolos transmitidos e usando o método em [6] para obter estimativas interpoladas da distorção nos intervalos de interesse [2].

\section{SIMULAÇÃO}

A função de densidade espectral de potência do processo simulado de desvanecimento de Rayleigh tem características de Butterworth de $3^{\mathrm{a}}$ ordem com largura de faixa de 3-dB, bi-lateral e normalizada pela taxa de símbolos $1 / \mathrm{T}$, denotada por BT. Em todos os resultados de simulação de taxa de erro de bit (BER), as curvas rotuladas de Ideal e de PSP correspondem à deteção com conhecimento ideal do desvanecimento e à deteção usando os algoritmos propostos neste artigo, respectivamente.

Nas Fig. 5.a e 5.b, para BT=.01 e BT=.05, respectivamente, são mostradas as curvas do sistema sem intercalação, com modulação 8-PSK codificada em treliça de 4 estados, sem transições paralelas e chamada doravante de Bat-4. As curvas rotuladas de BE são relativas ao método em [6], com uma treliça de decodificação expandida para 16 estados

A seguir, nos resultados de simulação do sistema com intercalação, as curvas rotuladas de Gen foram obtidas usando o algoritmo proposto neste artigo mas com conhecimento ideal das amostras da distorção nos intervalos de decodificação em que as estimativas não podiam ser obtidas do estimador de Kalman, devido ao problema de não-causalidade. Se o conhecimento ideal foi usado com o dobro da frequência necessária, as curvas resultantes são rotuladas de Gen2. Substituindo o conhecimento ideal pelas estimativas fornecidas pelo 


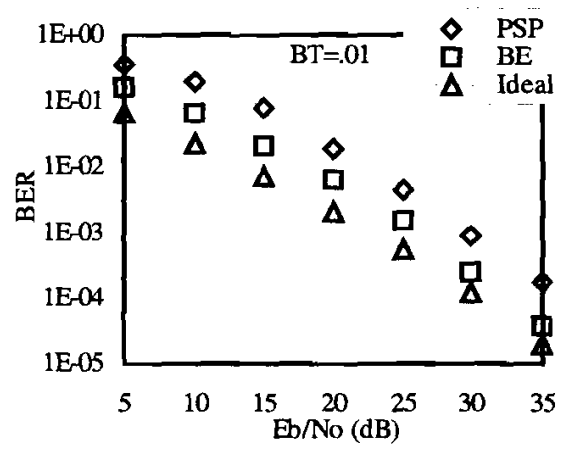

(a)

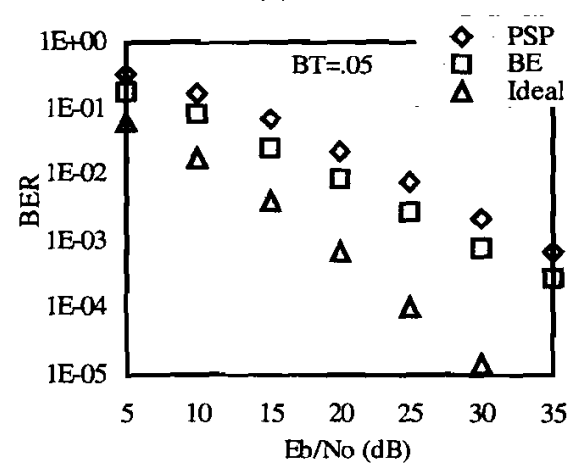

(b)

Fig. 5. Sistema sem intercalação, Bat-4 (a) $\mathrm{BT}=.01(\mathrm{~b}) \mathrm{BT}=.05$.

interpolador, foram obtidas as curvas PSP 〈PSP2 $\rangle$. As curvas rotuladas de $\mathrm{Pil}$ foram obtidas usando o método em [6] com a mesma taxa de símbolos conhecidos e o mesmo número de coeficientes de interpolação usados para obter as curvas PSP. Com exceção das curvas rotuladas de Ideal, todas as demais foram deslocadas para a direita para compensar a perda de energia com símbolos conhecidos. Nas Fig. 6.a e 6.b, são mostradas as curvas de simulação do sistema com intercalação usando modulação 8-PSK codificada em treliça com 8 estados, doravante chamada de Ung-8. As curvas são para $\mathrm{BT}=.05$ e bloco de intercalação 16×16. Na Fig. 6.a, a taxa de inserção de símbolos conhecidos é de $1: 9$ e o número de coeficientes do interpolador é 5; na Fig. 6.b, a taxa é de 1:17 e o número de coeficientes 3 .

Nas Fig. 7.a e 7.b, as curvas mostradas são para BT $=1$ e bloco de intercalação $8 \times 8$. Na Fig. 7.a, a taxa de inserção de símbolos conhecidos é de 1:5 e o número de coeficientes do interpolador é 5; na Fig. 7.b, a taxa é de 1:9 e o número de coeficientes é 3 . Esses resultados mostram que métodos que extraem observações de distorção apenas a partir de amostras do sinal recebido relativas a símbolos conhecidos, sofrem uma súbita queda de desempenho quando a taxa de inserção desses símbolos for menor que a taxa de Nyquist do processo de distorção. Como o método proposto neste

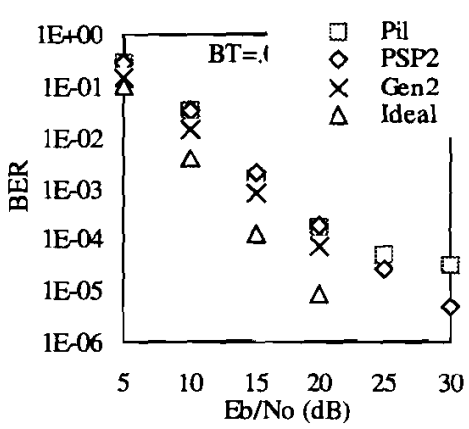

(a)

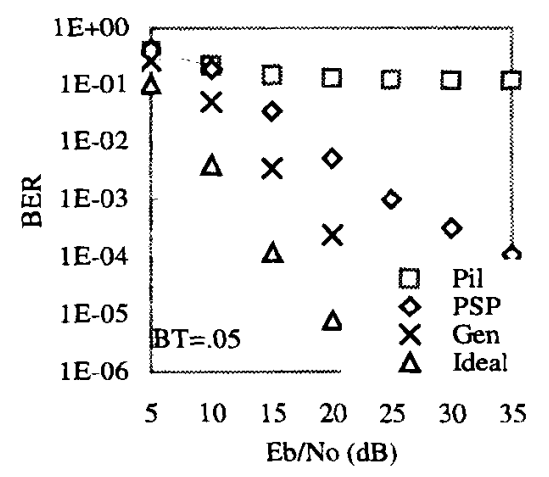

(b)

Fig. 6. Sistema com intercalação,Ung-8, BT=.05, bloco $16 \times 16$ (a) $\mathrm{L}=9, \mathrm{~K}=5$ (b) $\mathrm{L}=17, \mathrm{~K}=3$.

artigo também extrai observações das amostras relativas a símbolos de dados, a degradação do seu desempenho é mais suave.

Nas Fig. 8.a e 8.b são mostradas curvas obtidas das simulações do método proposto neste artigo e do método em [6], respectivamente, para sistemas com intercalação usando os códigos Bat-4, Ung-8 e Ung-16, este último se referindo à modulação 8-PSK codificada em treliça com 16 estados, sem transições paralelas. Os parâmetros da simulação foram $\mathrm{BT}=.1$, bloco de intercalação $8 \times 8$, taxa de símbolo conhecido de 1:5 e interpolador com 5 coeficientes. Os resultados na Fig. 8 mostram que, ao contrário do que acontece com o método que extrai observações da distorção a partir apenas de amostras relativas a símbolos conhecidos, o desempenho do método proposto neste artigo não sofre saturação com o uso de códigos mais poderosos, o que pode ser atribuído à redução de erros das decisões tentativas, resultando em estimativas melhores da distorção.

\section{REFERÊNCIAS}

[1] G.Ungerboeck, "Channel coding with multilevel/phase signals", IEEE Trans. Inform. Theory, vol. 28, pp. 5567, Jan. 82 


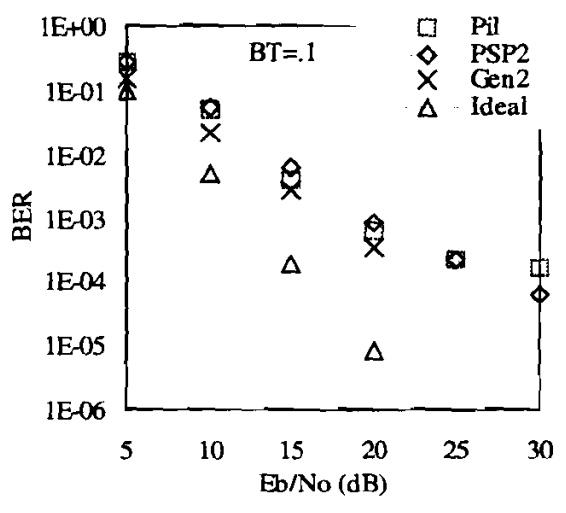

(a)

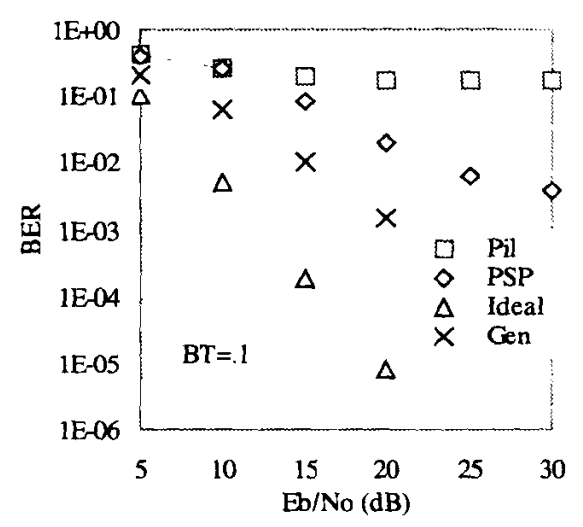

(b)

Fig. 7. Sistema com intercalação, Ung-8, BT=.1, bloco $8 \times 8$ (a) $L=5, K=5$ (b) $L=9, K=3$.

[2] T.Sugahara, "Deteção de sinais com Modulação Codificada em Treliça em canais com desvanecimento não-seletivo em frequência", Tese de Doutorado, Pontifícia Universidade Católica do Rio de Janeiro, Nov. 97.

[3] Divsalar, M. K. Simon, "The design of Trellis Coded MPSK for fading channels: performance criteria", IEEE Trans. Commun., vol. 36, no. 9, pp.1004-1012, Sep. 88.

[4] K. Cavers, P. Ho, "Analysis of the error performance of Trellis-Coded Modulations in Rayleigh fading channels", IEEE Trans. Commun., vol. 40, no. 1, pp.74-83, Jan. 92.

[5] R.van Novelen, D.P.Taylor, "Analysis of the pairwise error probability of noninterleaved codes on the Rayleigh fading channel", IEEE Trans. Commun., vol. 44, no. 4, pp.456-463, Apr. 96.

[6] K. Cavers, "An analysis of pilot symbol assisted modulation for Rayleigh fading channels", IEEE Trans. Vehicular Technol., vol. 40, pp.686-693, Nov.91.

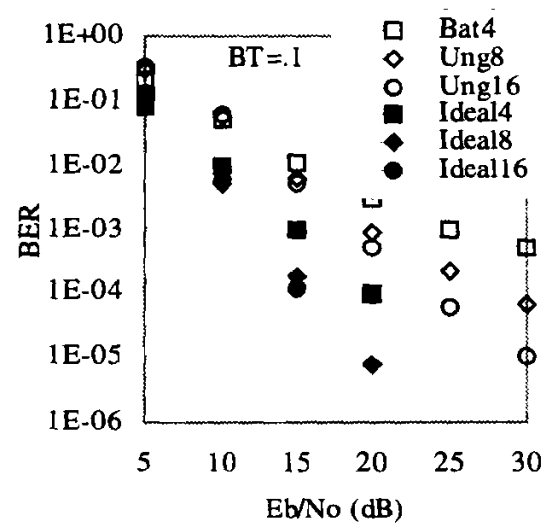

(a)

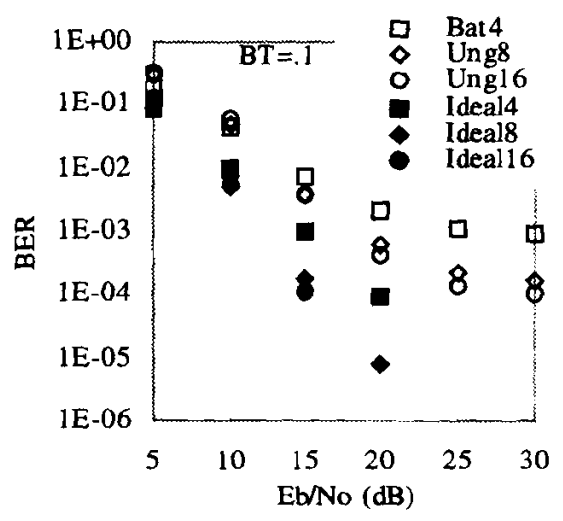

(b)

Fig. 8. Efeito do código, bloco $8 \times 8, \mathrm{~L}=5, \mathrm{~K}=5$ (a) método deste artigo (b) método em [5].

[7] M. Vitetta and D. P. Taylor, "Maximum likelihood decoding of uncoded and coded PSK signal sequences transmitted over Rayleigh flat-fading channels", IEEE Trans. Commun.,vol.43,no.11, pp. 2750-2758, Nov.95.

[8] N. D'Andrea, A. Diglio and U. Mengali, "Symbolaided channel estimation with nonselective Rayleigh fading channels", IEEE Trans. Vehicular Technol., vol.44, no.1, pp. 41-49, Feb.95.

[9] P. Y. Kam and H. M. Ching, "Sequence estimation over the slow nonselective Rayleigh fading channel with diversity reception and its application to Viterbi decoding", IEEE J. Select. Areas Commun., vol. 10, pp. 562-570, Apr.1992.

[10] Polydoros and R. Raheli, "The Principle of PerSurvivor Processing: a general approach to approximate and adaptive ML sequence estimation",Technical Report, CSI-90-07-05, USC, Jul.90.

Tomie Sugahara received the B.Sc and the M.Sc. degrees in 1975 and 1977, respectively, both from the Universidade Federal do Rio de Janeiro, the Engineer degree in 1984 from 
the University of California, Los Angeles, and the Ph.D. degree in 1997 from the Pontifícia Universidade Católica do Rio de Janeiro, all in Electronic Engineering. From 1986 to 1993 she worked in electronic and communication industry, where she was involved with digital radio equipment design and test. Her research interests include coding, estimation and detection techniques for mobile communications.

Raimundo Sampaio Neto nasceu em Rezende, RJ, em 16 de junho de 1952. Formou-se em Engenharia Elétrica (Telecomunicações) na Pontifícia Universidade Católica do Rio de Janeiro (PUC-Rio) em 1975 e obteve o grau de Mestre em Ciências em Engenharia Elétrica na mesma universidade em 1978. Concluiu o doutorado na University of Southern California (USC) em Los Angeles em 1983, com o título de Ph.D em Engenharia Elétrica. De 1983 a 1984 prestou serviços de consultoria em análise de sistemas de comunicações junto à Axiomatic Inc., em Los Angeles, e desenvolveu atividades de pesquisa na área de sincronização de códigos em sistemas spread spectrum, como Postdoctoral Fellow do Communication Sciences Institute da USC. Em julho de 1984 retornou à PUC-Rio onde é atualmente Professor Associado, trabalhando em ensino e pesquisa no Centro de Estudos em Telecomunicações da Universidade Católica (CETUC). Durante o ano de 1991, enquanto em licença sabática da PUC-Rio, foi Professor Visitante do Departamento de Engenharia Elétrica da USC. Suas principais áreas de interesse são comunicações via satélite, sincronização em sistemas digitais e processamento digital de sinais. 